\title{
Emotion does not influence prefrontal cortex activity during a visual attention task. A functional near- infrared spectroscopy study.
}

\author{
Robert C. A. Bendall and Catherine Thompson \\ Directorate of Psychology and Public Health \\ University of Salford \\ Salford, United Kingdom \\ r.c.a.bendall@salford.ac.uk
}

\begin{abstract}
Research shows that positive and negative emotion can influence a range of cognitive processes as well as hemodynamic prefrontal cortex (PFC) activity. This study sought to investigate if PFC activity was influenced by positive and negative stimuli during mood induction as well as during a subsequent visual attention task. The International Affective Picture System was employed to induce affective states in participants before they completed a visual attention task. PFC hemodynamic activity was recorded using non-invasive functional near-infrared spectroscopy. Increased PFC activity was evident during the visual attention task compared to affective stimuli viewing. However, emotion did not influence PFC activity during affective stimuli viewing or completion of the visual attention task. Future research should take into account the role individual differences may play in mitigating any influence of emotion on PFC activity.
\end{abstract}

Emotion; fNIRS; PFC; visual attention; mood; cognition

\section{INTRODUCTION}

Recently there has been increased focus regarding the influence of emotion on cognition at both the behavioural and neural level. At the behavioral level emotion has been shown to influence multiple aspects of cognition including working memory [1], task shielding [2], attentional control [3], and visual attention $[4,5,6]$. At the neural level research has shown that the prefrontal cortex (PFC) is involved in emotional processing during cognitive task completion; see [7, 8] for recent compressive reviews of this research area.

Information processing models highlight the importance of both top-down and bottom-up processing during cognitive task completion. For example, within the field of visual attention researchers propose that top-down processing is characterized by goal-directed behavior whereas bottom-up processing refers to the automatic capture of attention by salient information in the environment regardless of task demand [9]. It has been argued that during tasks investigating the effects of emotion on cognition it is likely that both processing strategies are evident [7]. For instance during cognitive tasks involving emotional distractors, it is suggested that the influence of the emotional distraction depends on interactions between neural systems that allow an individual to stay focused on the task as well as systems responsible for the processing of emotional information [7]. It is proposed that these two neural systems compete for processing resources and emotional distractors result in bottom-up processing of task-irrelevant information. This increase in bottom-up processing is likely to have a detrimental influence on task performance. Indeed evidence shows that negative mood is correlated with poorer performance during a working memory task incorporating emotional distractors [1]. It has been argued that impairments in task performance can be mitigated by employing top-down cognitive control processes. These processes allow systems involved in the regulation of emotional responses to be activated and therefore deal with any emotional distraction present [7].

To assume that effective top-down cognitive control processes are required for effective functioning in cognitive tasks leads to an important observation in clinical and affective populations. It has been reported that healthy individuals perform better than clinical populations in tasks involving executive control [10]. Further, it has been suggested that difficulty in utilizing top-down control processes to regulate emotion is a contributing factor in various clinical and affective disorders including major depressive disorder (MDD) [10, 11], bipolar disorder (BD) [10] and borderline personality disorder $[12,13]$. Research investigating the influence of emotion on cognition in both healthy and clinical populations will advance our understanding of the impact of emotion on cognition.

Research that explores the effects of emotion on cognition at the neural level often concentrates on the role of the amygdala. The amygdala is a brain structure located deep in the brain involved with the processing of emotional information. It has been suggested that emotion regulation encompasses neural activation resulting from interactions between the hot emotional system found in limbic regions (e.g. the amygdala) and the cold, higher order emotional systems found in prefrontal regions [7, 14]. According to the limbic model the amygdala forms part of the evolutionary old brain found in sub-cortical regions, and is involved in the generation of primitive emotions (e.g. fear). Emotions are then elaborated in the advanced PFC neural circuits. 
Anatomically there are both direct and indirect pathways connecting the hot and cold emotional systems. For instance it has been shown that the ventral medial prefrontal cortex (vmPFC) is heavily innervated by the amygdala [15]. The medial prefrontal cortex (mPFC), which includes both the vmPFC and the dorsomedial prefrontal cortex (dmPFC), is heavily involved in the processing of emotional and social stimuli [15]. Further, the vmPFC has connections with the anterior cingulate, dorsolateral prefrontal cortex (dlPFC) and ventro lateral prefrontal cortex (vlPFC). These anatomical connections link medial brain areas involved in the processing of emotional information with lateral/dorsal brain regions implicated in higher order executive function [15]. Additionally the dlPFC has been cited as receiving influencing effects from the amygdala via indirect pathways through the cingulate and posterior orbitofrontal cortex [16].

Given the emerging role of the PFC during emotional processing it is important to study the influence of emotion on PFC activation. Functional near-infrared spectroscopy (fNIRS) is a novel, non-invasive neuroimaging technique that allows the study of PFC activation. fNIRS measures hemodynamic concentration changes in the brain similar to functional magnetic resonance imaging (fMRI). Whereas fMRI measures the paramagnetic properties of deoxygenated-hemoglobin (deoxy-Hb) to infer neural activity, fNIRS uses the differing light absorption properties of oxygenated-hemoglobin (oxy$\mathrm{Hb})$ and deoxy-Hb in the near-infrared range $(650-1000 \mathrm{~nm})$ to infer neural activation changes. The relationship between vascular response and neural activity is termed neurovascular coupling and is described in detail in [17]. Research has shown that the fMRI blood oxygen level dependent (BOLD) signal and fNIRS signals are correlated suggesting that studies using fMRI and fNIRS can be compared [18]. One of the limitations of fNIRS is that it can only measure hemoglobin concentration changes in the cortex at a depth of $1-2 \mathrm{~cm}$. However, fNIRS also has many benefits including its quick application time, non-invasiveness, portability, and ability to be used in more ecologically valid testing environments. Moreover, it has been suggested that fNIRS is a suitable neuroimaging methodology for investigating emotion-cognition interactions in the PFC [19, 20].

Recent fNIRS research has helped in understanding the important role that the PFC has regarding the impact of emotion on cognition. It has been demonstrated that emotional stimuli influences oxy- $\mathrm{Hb}$ activity in the mPFC during an nback task measuring attentional control [3]. With a compound measure of oxygenation (oxy-Hb - deoxy-Hb), [21] provided evidence for the involvement of the medial rostral prefrontal cortex (mrPFC) during emotional introspection and identification of positively valenced stimuli (visual art) compared to neutral stimuli. In addition, [1] have shown that naturalistic mood (mood occurring naturally in individuals as opposed to experimentally induced mood or that observed in clinical and affective populations) is correlated with PFC activity during a working memory task. Here individuals with high levels of negative mood displayed lower levels of PFC activity during the task. This observation is important as it demonstrates that emotion has an influence on cognition at the neural level in everyday situations. Differences in PFC oxy-Hb activity have also been observed in MDD and BD patients [10].
In an emotional Stroop task differing patterns of PFC oxy-Hb activity were observed in BP patients and MDD patients (in remission) compared to healthy individuals. Additionally, varying patterns of neural activity in response to positive stimuli was also evident in BP and MDD patients suggesting that different neural circuitry is involved during emotional processing in these two disorders [10]. The aforementioned fNIRS research conducted on healthy individuals and clinical populations, combined with the observation that fNIRS signals are correlated with the fMRI BOLD response, suggest that fNIRS is a suitable neuroimaging tool for investigating the neural correlates of the relationship between emotion and cognition within the PFC.

Despite this past research, the exact role of the PFC within this relationship is not clear and differences in behavioural and neuroimaging data have been observed within the published literature. For example, behavioural data has reported that emotion does influence cognition [6], whilst other data suggest this is not the case [22]. Similarly, neuroimaging data have reported increased activity in the mrPFC for positive but not neutral stimuli [21] whereas [3] observed increased activity during viewing of both negative and neutral images. An increase in oxy-Hb activity for negative stimuli (compared to neutral) has also been observed by [23]. In addition to findings revealing different patterns of PFC activity in response to viewing emotional stimuli, studies also show differences in activity within the same cognitive task. For example, it has been shown that lower levels of PFC activity during a working memory task are evident for individuals with higher levels of negative mood [1], whereas [3] have demonstrated higher levels of activation during a working memory task after exposure to negative but not neutral stimuli. Moreover, recent evidence has shown that task difficulty can moderate the influence of emotion on PFC activity. Here it was shown that in the multi-source interference task threat distractors decreased activity in the dlPFC during difficult trials, whereas they increase activity during easy trials. This finding suggest interactions are evident in brain regions involved in cognitive control as dlPFC activity in response to threat distractors differ according to task difficulty [24]. The study of the influence of emotion on cognition at the neural level warrants further investigation.

The investigation of the way in which emotion influences cognition has progressed recently with researchers exploring additional aspects of neural processing during cognitive tasks. The current study will identify the effect of emotion on cognition at the neural level with the aim of explaining some of the conflicting findings within this field. Given the importance of the PFC in emotion and cognition the study will utilize fNIRS to measure changes in activation in this brain region. The change detection flicker paradigm [25], which measures visual attention, will be employed. Change blindness is the failure to detect changes in a visual scene due to a brief interruption that prevents bottom-up capture of attention by the motion transient that usually accompanies a change. The detection of a change in the visual environment is therefore highly dependent upon top-down processing. Currently there is no published research examining whether mood influences PFC activity during a change detection flicker task. 
The study aimed to investigate 1) how PFC activity varies according to emotional state, and 2) if emotion effects PFC activation in a cognitive task requiring significant top-down processing. It was predicted that positive mood would increase PFC activity whereas negative mood would decrease PFC activity during both the mood induction stage and the visual attention task. It was also predicted that PFC activity would be greater during the visual attention task compared to during the mood induction task. Therefore, we predict a main effect of emotion, a main effect of task, and no interaction effects.

\section{METHOD}

\section{A. Participants}

Thirty two healthy individuals (20 female) aged between 18 and $38(M=24.8$ years; $S D=5.4$ years $)$ participated in this study. Written informed consent was obtained from each participant after receiving procedural information regarding the study. Ethical approval was obtained from the College of Health and Social Care Ethics Panel at the University of Salford. All participants received a $£ 10$ inconvenience allowance.

\section{B. Design}

A within-participants design was used with two independent variables; mood (positive, neutral, or negative) and task (emotional stimuli viewing task and visual attention task). The dependent variable was changes in oxy-Hb. A measure of positive and negative affect was also recorded to validate the method of inducing emotion.

\section{Materials and apparatus}

\section{1) Emotion induction / affective stimuli}

Emotion was manipulated by presenting participants with visual images of differing emotional valence from the International Affective Picture System (IAPS) [26]. 60 images were selected; 20 positive (mean valence 7.65 , mean arousal 5.05), 20 neutral (mean valence 4.62, mean arousal 3.11), and 20 negative (mean valence 2.35 , mean arousal 5.17) images. A further 20 positive images were shown at the end of the experiment (mean valence 7.87, mean arousal 5.13). All images were presented in color and measured a maximum of 1024 x 768 pixels. The Positive and Negative Affect Schedule (PANAS) [27], a 20-item self-report measure of positive and negative affect, was used to record participants' affect after presentation of affective stimuli. The PANAS consists of 20 words that describe positive and negative feelings and emotions. For each word participants were asked to "indicate to what extent you feel this way right now, that is, at the present moment' on a scale of 1 (very slightly or not at all) to 5 (extremely). The PANAS provides a measure of positive affect from the summed rating of all positive words and a measure of negative affect from the summed rating of the negative words. The minimum score for each measure is 10 (indicating low affect) and the maximum is 50.

\section{2) fNIRS data collection}

In order to record cortical PFC oxy-Hb an fNIR Imager 1000 (fNIR Devices, LLC.) multichannel fNIRS system was used. This system has a temporal resolution of $500 \mathrm{~ms}(2 \mathrm{~Hz})$ and consists of 4 light sources and 10 light detectors resulting in 16 measurement optodes. The probe was placed on the forehead aligned to frontal polar (Fp) 1 and Fp2 of the International 10-20 system [28] where Fpz corresponded to the midpoint of the probe (Figure 1) [29]. Changes in oxy-Hb were recorded with Cognitive Optical Brain Imaging Studio (fNIR Devices, LLC.) and analyzed offline using fnirSoft [30]. Raw data was processed using a finite impulse response linear phase low-pass filter, with order 20 and cut-off frequency of $0.1 \mathrm{~Hz}$, to attenuate high frequency noise, respiration, and cardiac effects. Additionally a sliding-window motion artifact rejection algorithm was employed to remove motion artifacts and saturated channels. These methods are described in detail in [29]. Optodes were also visually inspected for signs of light saturation or light obstruction caused by hair beneath the sensor. Oxy-Hb was then calculated using the modified BeerLambert Law [31]. Three scene viewing and three visual attention task data segments were extracted using synchronization markers received from E-Prime (Psychological Software Tools, Inc) and averaged for statistical analysis.

\section{Procedure}

After providing written informed consent participants were seated approximately 22 inches from the screen. The fNIRS sensor was placed on a participant's forehead and secured using elastic strapping. The emotion induction images and visual attention task were presented on a computer using EPrime (Psychological Software Tools, Inc) with a 19 inch monitor. Participants completed 3 blocks of trials and block order was counterbalanced. In each block, 20 color photographs taken from the IAPS were shown for $5000 \mathrm{~ms}$ each in random order and were followed by a 500ms interstimulus interval (ISI). Images were neutral, positive, or negative, depending on block order. After presentation of the

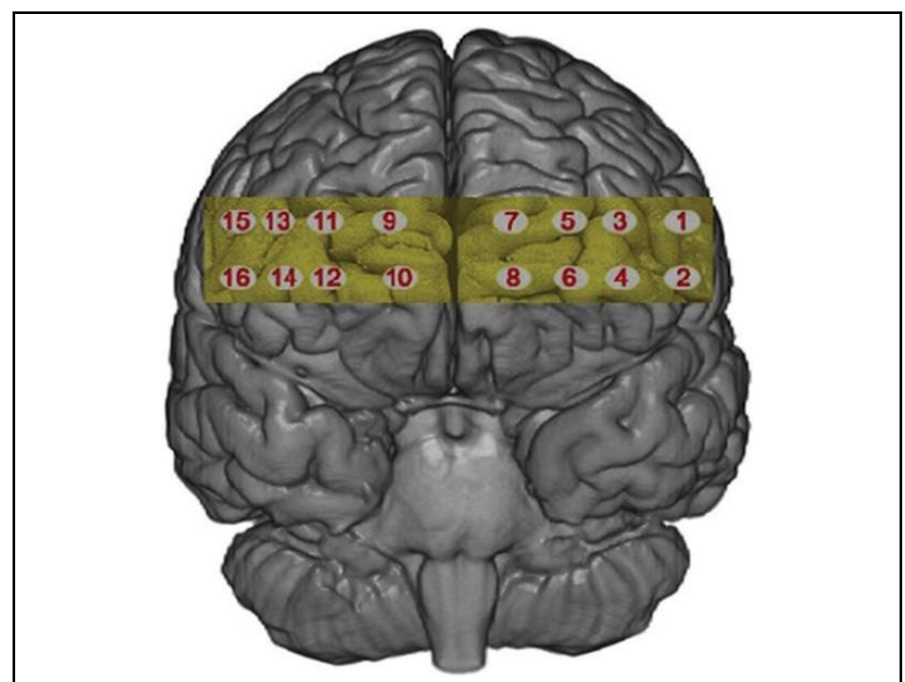

Figure 1. fNIRS probe location showing 16 optode sites on the prefrontal cortex. 
images participants completed the PANAS followed by the change detection task. During a trial an image of neutral valence was presented for $1000 \mathrm{~ms}$ followed by a blank image for $500 \mathrm{~ms}$. A changed image, which comprised the original image with a subtle change, was then presented for $1000 \mathrm{~ms}$ followed by another blank image for 500ms. This cycle repeated until the participant identified the change at which point they pressed the space bar on the keyboard (Figure 2). After completion of the final change detection block participants were presented with 20 additional positive images from the IAPS. This was intended to make certain that participants were induced into a positive mood when leaving the lab. Participants were then thanked for their time and given $£ 10$ as an inconvenience allowance.

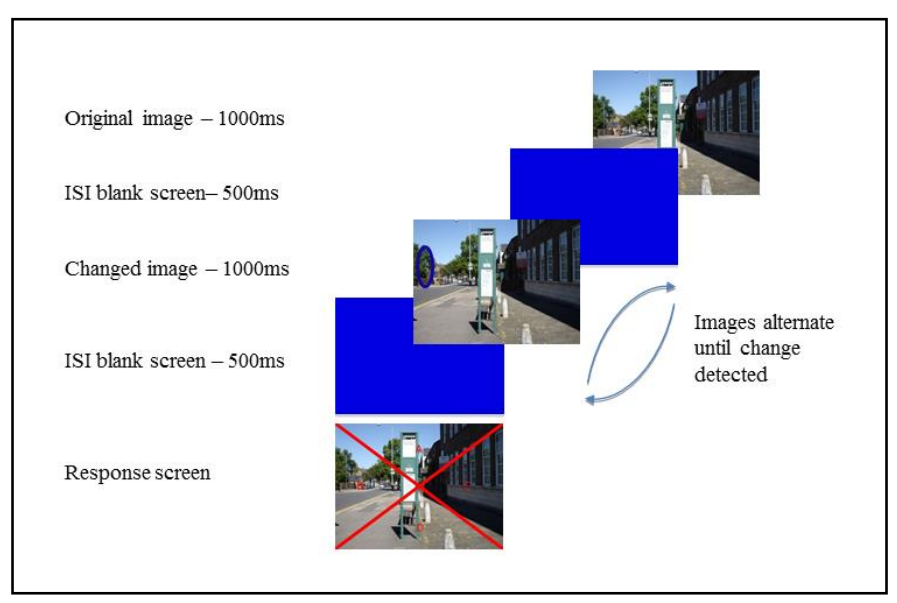

Figure 2. Illustration of the change detection flicker task.

\section{RESULTS}

A. Affect scores

Two one-way repeated measures analysis of variance (ANOVA) followed by planned comparisons were conducted to check that the affective stimuli were successful at inducing positive and negative mood states in participants.
Analysis of the positive affect scores showed a significant effect of mood, $F(2,60)=21.782, M S E=29.161, p<.001$. Planned comparisons revealed that viewing positive stimuli significantly increased positive mood scores compared to neutral stimuli $(29.4$ vs. $20.5 ; F(1,30)=33.610, M S E=$ $72.583, p<.001$; Figure 3 ). Higher positive mood scores were also evident after viewing negative (23.4) compared to neutral stimuli, $F(1,30)=7.290, M S E=35.049, p<.05$; Figure 3).

Analysis of negative affect revealed that viewing emotional stimuli influenced negative mood, F $(2,60)=40.441, M S E=$ $15.488, p<.001$. Planned comparisons showed that viewing negative stimuli significantly increased negative mood scores compared to neutral stimuli $(21.4$ vs. $14.8 ; F(1,30)=41.024$, $M S E=33.045, p<.001$; Figure 3). Higher negative mood scores were also evident after viewing neutral compared to positive stimuli $(14.8$ vs. $12.8 ; F(1,30)=4.647, M S E=$ 25.832, $p<.05$; Figure 3).

\section{B. fNIRS results}

Analysis for each optode was conducted separately resulting in sixteen 2 (task) x 3 (emotion) repeated measures ANOVAs that investigated the effects of task and emotion on oxy-Hb in the PFC. Significant main effects of task were evident in optodes $1-3,5,7-9,11$, and 12 demonstrating higher levels of oxy- $\mathrm{Hb}$ during change detection trials compared to stimuli viewing (Figure 4; Table 1). No significant effect of task was evident in optodes 6, 13-16. Optodes 4 and 10 displayed a trend towards increased oxy- $\mathrm{Hb}$ activity during change detection trials compared to the stimuli viewing task (Table 1). There were no significant effects of emotion on oxy$\mathrm{Hb}$ activity in any of the optodes and no significant interaction between emotion and task (Table 1).

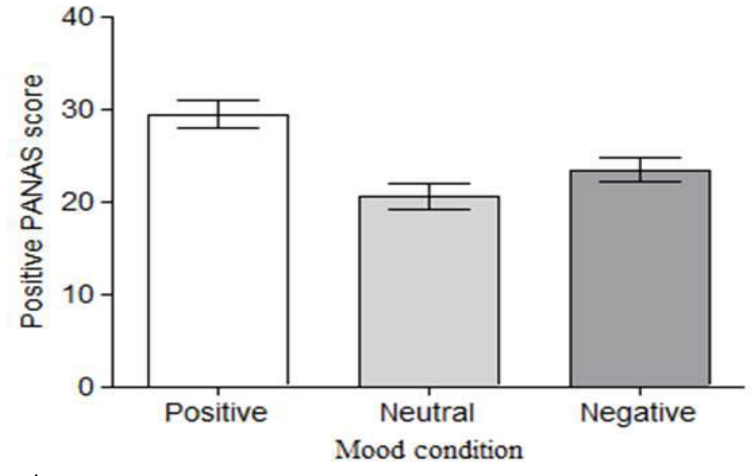

$\mathbf{A}$

Figure 3.

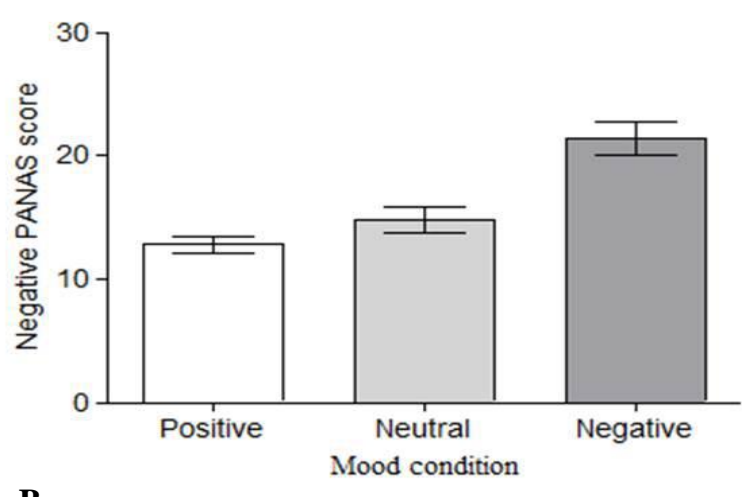

B Mean PANAS scores for each induced mood condition.
Self-reported mood varied across the conditions for both
positive affect (A) and negative affect (B). Error bars =
standard error of the mean. 


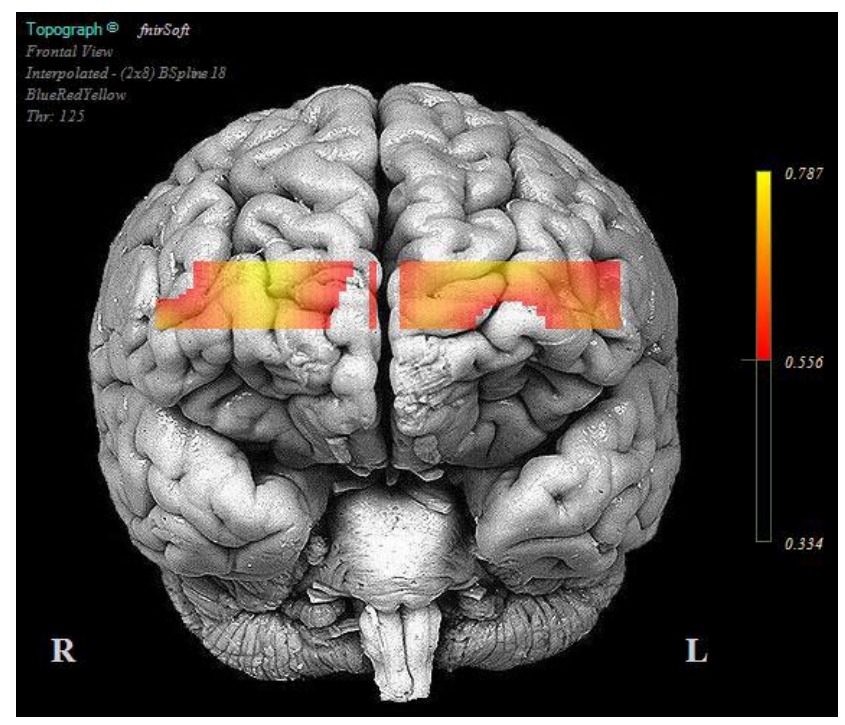

Figure 4. Increased oxy-Hb activation during the change detection task compared to scene viewing across the PFC. The increase in PFC activation during the change detection task compared to stimuli viewing task is visualized by subtracting stimuli viewing oxy-Hb activation from change detection task oxy-Hb activation.

\section{DISCUSSION}

The aim of the current study was to investigate the underlying PFC activity during the viewing of emotional stimuli and during a subsequent cognitive task. It is the first study to use the change detection flicker task to investigate the influence of emotion on hemodynamic activity in the PFC (during a visual attention task). The exact role of the PFC in the relationship between emotion and cognition is still unknown despite recent advances in this area. Current research reports conflicting results in terms of PFC neural activity during the viewing of emotional stimuli $[3,21]$ as well as during cognitive task performance after emotion induction [1, 3]. Further investigation in this area will help to identify the role of the PFC in healthy populations, as well as inform research exploring emotional processing in clinical and affective populations.

Higher PFC oxy-Hb activity in 9 out of 16 optodes during the change detection task compared to the stimuli viewing task (irrespective of emotion condition) most likely demonstrates the increased cognitive resources needed to complete the change detection task. However, the neuroimaging data suggest that PFC activity did not differ between emotion conditions indicating that emotion does not influence PFC oxy-Hb activity during the viewing of emotion inducing stimuli or during a

TABLE 1. RESUlTS OF 3 (EMOTIONAL VALENCE) X 2 (COGNITIVE

TASK) REPEATED MEASURES ANOVAS OF OXY-HB

CONCENTRATION CHANGES.

\begin{tabular}{|c|c|c|c|c|c|c|c|c|c|}
\hline \multirow[t]{2}{*}{ Channel } & \multicolumn{3}{|c|}{ Emotional valence } & \multicolumn{3}{|c|}{ Task } & \multicolumn{3}{|c|}{ Interaction } \\
\hline & $F$ & $M S E$ & $p$ & $F$ & $M S E$ & $p$ & $F$ & $M S E$ & $p$ \\
\hline 1 & 1.052 & 3.525 & .362 & 8.977 & 1.290 & $.009 * *$ & 1.773 & 1.057 & .198 \\
\hline 2 & .798 & 4.299 & .455 & 7.244 & 2.174 & $.012 *$ & .331 & 1.837 & .720 \\
\hline 3 & .596 & 5.495 & .555 & 9.170 & 2.236 & $.006^{* *}$ & .806 & 1.422 & .453 \\
\hline 4 & .773 & 3.147 & .468 & 3.561 & 2.320 & .073 & 1.008 & 1.504 & .373 \\
\hline 5 & $\begin{array}{l}.089 \\
\end{array}$ & 3.551 & .915 & 12.130 & 1.339 & $.002 * *$ & .191 & 1.033 & .827 \\
\hline 6 & .244 & 4.552 & .784 & 2.096 & 2.528 & .159 & .141 & 2.061 & .869 \\
\hline 7 & .406 & 3.863 & .669 & 8.032 & 2.622 & $.009 * *$ & .104 & 1.132 & .901 \\
\hline 8 & .342 & 3.468 & .712 & 6.466 & 3.058 & $.017 *$ & .092 & 1.939 & .912 \\
\hline 9 & .206 & 3.405 & .815 & 4.290 & 2.535 & $.049 *$ & .168 & .939 & .846 \\
\hline 10 & .327 & 4.839 & .723 & 3.307 & 3.151 & .082 & .513 & 2.283 & .602 \\
\hline 11 & .230 & 3.016 & .795 & 13.815 & 1.299 & $.001 * *$ & .543 & 1.584 & .585 \\
\hline 12 & .096 & 7.455 & .909 & 9.457 & 2.477 & $.005 * *$ & .490 & 1.808 & .615 \\
\hline 13 & 1.255 & 5.132 & .302 & 1.246 & 3.621 & .285 & .305 & 3.840 & .739 \\
\hline 14 & .740 & 6.248 & .491 & .719 & 4.017 & .418 & .286 & 2.612 & .755 \\
\hline 15 & .270 & 5.976 & .767 & 1.953 & 1.953 & .479 & .575 & 1.637 & .574 \\
\hline 16 & .238 & 5.296 & .789 & 1.858 & 2.503 & .192 & .139 & 1.569 & .870 \\
\hline
\end{tabular}

\footnotetext{
$* p<.05 ; * * p<.01$
} 
subsequent cognitive task. Additionally, no interaction effect between emotion group and task group was observed.

The observation of increased PFC oxy-Hb during the visual attention task compared to the emotional stimuli viewing task is indicative of increased cognitive demand during the visual attention task. The stimuli viewing task was passive, possibly involving bottom-up processing, whereas the visual attention task was more demanding and required additional top-down processing. Previous research utilizing fNIRS has shown increases in PFC oxy-Hb activity as a cognitive task gets progressively more difficult $[32,33]$. Despite these differences in oxy-Hb between our two tasks no influence of emotion on PFC oxy-Hb activity was observed.

The finding that viewing different types of emotional stimuli has no impact on PFC oxy-Hb activity was surprising as previous research has demonstrated emotion-related changes in PFC oxy-Hb activity $[3,10]$. One possible explanation could be that participants in the current study were not successfully induced into the experimental mood conditions. However, the results from the self-report PANAS suggest that emotional stimuli did induce positive and negative emotion in participants. In relation to mood induction during the cognitive task, due to the fact that our PANAS measures were completed immediately after the viewing of affective stimuli, rather than after (or in addition to) the change detection task had been completed, we cannot rule out the possibility that participants were induced into mood groups after viewing affective stimuli only for their mood to return to normal as they completed the change detection task. One possible reason could be the fact that the stimuli used in the change detection task were controlled so that all images were of neutral affect. Research has previously shown that the influence of emotion on cognition can be dependent upon the valence of the specific task stimuli used [3]. It is worth noting here that we did observe an unexpected finding when analyzing the PANAS data. Participants reported higher positive mood scores after viewing negative compared to neutral stimuli. One possibility is that participants were responding to increased arousal in the negative images compared to neutral images. The important finding regarding the negative mood PANAS data is consistent with expectations that after viewing negative stimuli higher levels of negative mood were reported validating our mood induction method.

The non-significant effect of emotion on PFC oxy-Hb activity was also unexpected and this may be due to the way in which data is analyzed. For example, in the current study emotion-related oxy-Hb changes were compared against each other (positive, neutral, and negative mood) and no differences in activity were observed. In contrast, in [3] changes in oxy-Hb for neutral and negative stimuli were compared against baseline oxy-Hb activation and it was shown that both neutral and negative stimuli increased PFC activation. When oxy-Hb activity was directly compared across neutral and negative stimuli conditions no differences in activation were evident. This finding is similar to the observation in the current study and suggests that comparing oxy-Hb changes to baseline data may inflate differences in activation.

The lack of any emotion-dependent changes in PFC oxy-Hb activity during the change detection task could be due to the methodological issue concerning the stimuli valence described above. However, previous research has shown that naturally occurring negative mood can influence PFC oxy-Hb activity during a cognitive task [1]. Additionally, it has also been demonstrated that oxy- $\mathrm{Hb}$ activity during a cognitive task is increased after participants viewed negative emotional stimuli compared to neutral stimuli [3]. It would be interesting to see if there are interactions between the difficulty level of the visual attention task employed and emotion on behavioral performance and underlying neural PFC activity. Recent evidence has shown that threat distractors can decrease activity in the dlPFC during demanding trials in a multi-source interference task, whereas they increase activity during less demanding trials (compared to no distractor trials) [24]. This finding suggests such interactions may be evident in brain regions involved in cognitive control as changes in dlPFC activity in response to threat distractors differ according to task difficulty. It has also been shown that PFC oxy-Hb activity increases as a cognitive task becomes more demanding [32, 33]. It is possible that as the current study only adopted one level of task difficulty the task may have been too demanding and therefore masked any possible influence of emotion on $\mathrm{PFC}$ oxy-Hb activation. However, if this was the case we would still expect to observe emotion-related changes in PFC oxy-Hb activity during the passive stimuli viewing task which we did not observe.

Cognitive theories attempt to explain behaviour in terms of accurate fits with average group-level performance. However, these models often fit less accurately when they are applied to individuals [34]. Individual differences are likely to mitigate the effect of emotion on cognition and evidence from healthy populations supports this view. For example, it has been shown that neuroticism modulates amygdala-PFC connectivity in response to negative emotional facial expressions [35] Interestingly, neuroticism and extraversion have been shown to predict attentional performance during change detection [36] Additionally, [37] have shown that state negative affect and trait negative affect have both combined and separate influences on attentional processing. Further, it has been shown that in individuals with experience of early-life stress, personality traits linked to emotional control are related to sustained vmPFC activity during a mildly stressful task. The vmPFC is involved in emotional processing and regulation of amygdala activity [38]. Finally, in a study investigating the influence of negative images and the positive affect trait on amygdala activation, it was found that amygdala response was influenced by both task demand and the positive affect trait [39]. These findings demonstrate that individual differences play a role in mitigating the effect of emotion on cognition.

The current study did not take into account any individual differences in mood/depressive symptoms or personality traits and these need to be investigated further. Initial research has demonstrated that rumination and mindfulness are linked to vmPFC activity involved in regulating amygdala activity [38] and are likely to moderate the effect of emotion on cognition. In addition, attentional impulsiveness (an index of impaired executive function) has been shown to be correlated with amygdala and dlPFC activity, both of which are brain regions involved in emotional and executive functioning during emotional distraction [40]. It is likely that attentional 
impulsiveness will also impact upon the manner in which emotion influences cognition at both the behavioural and neural level. Research investigating individual differences in mood and cognition is important as it will help to advance our understanding of the brain regions and neural correlates in healthy individuals as well as those individual factors that may increase susceptibility to affective disorders.

In conclusion, using fNIRS there was no evidence that mood influences PFC oxy-Hb activity during affective stimuli viewing or during a subsequent visual attention task. This was unexpected and conflicted with the hypothesis as changes in oxy-Hb in response to emotional stimuli have been documented previously. It is possible that participants were not successfully induced into emotion groups, or that differences in the current experimental design and analysis and those used in past research influenced any potential observable differences. The finding of increased PFC oxy-Hb activity during the visual attention task compared to the emotional stimuli viewing task suggests the former is more cognitively demanding. This task requires additional top-down processing compared to the more passive (and bottom-up) stimuli viewing task. Recent research also suggests that individual differences mitigate the effects of emotion on cognition at the neural level. There is scope to expand on this work to investigate whether such influences have contributed to the current findings.

\section{ACKNOWLEDGMENT}

The authors would like to thank Dr Peter Eachus and Professor David Roberts for constructive comments.

\section{REFERENCES}

[1] Aoki, R., Sato, H., Katura, T., Utsugi, K., Koizumi, H., Matsuda, R., and Maki, A. (2011). Relationship of negative mood with prefrontal cortex activity during working memory tasks: An optical topography study. Neuroscience Research, 70, 189-196.

[2] Zwosta, K., Hommel, B., Goschke, T., and Fisher, R. (2013). Mood states determine the degree of task shielding in dual-task performance. Cognition and Emotion, 27(6), 1142-1152.

[3] Ozawa, S., Matsuda, G., and Hiraki, K. (2014). Negative emotion modulates prefrontal cortex activity during a working memory task: a NIRS study. Frontiers in Human Neuroscience, 8(46), 1-10.

[4] Fredrickson, B. L., and Branigan, C. (2005). Positive emoitions broaden the scope of attention and thought-action repitoires. Cognition and Emotion, 19(3), 313-332.

[5] Rowe, G., Hirsh, J. B., and Anderson, A. J. (2007). Positive affect increases the breadth of attentional selection. Proceedings of the National Academy of Sciences of the United States of America, 104, $383-$ 388.

[6] Wadlinger, H. A., and Isaacowitz, D. M. (2006). Positive mood broadens visual attention to positive stimuli. Motivation and Emotion, 30(1), 87-99.

[7] Dolcos, F., Iordan, A. D., and Dolcos, S. (2011). Neural correlates of emotion-cognition interactions: A review of evidence from brain imaging investigations. Journal of Cognitive Psychology, 23(6), 669694.

[8] Dolcos, F., Wang, L., and Mather, M. (2014). Current research and emerging directions in emotion-cognition interactions. Frontiers in Integrative Neuroscience, 8(83), 1-11.

[9] Itti, L., and Koch, C. (2000). A saliency-based search mechanism for overt abd covert sifts of visual attention. Vision Research, 40(10-12), 1489-1506.

[10] Matsubara, T., Matsuo, K., Nakashima, M., Nakano, M., Harada, K., Watanuki, T., ... Watanabe, Y. ( 2014). Prefrontal activation in response to emotional words in patients with bipolar disorder and major depression disorder. Neuroimage, 85, 489-497.

[11] Johnstone, T., van Reekum, C. M., Urry, H. L., Kalin, N. H., and Davidson, R. J. (2007). Failure to regulate: counterproductive recruitment of top-down prefrotnal-subcortical cicuitry in major depression. Journal of Neuroscience, 27(33), 8877-8884.

[12] Krause-Utz, A., Oei, N. Y. L., Niedtfeld, I., Bohus, M., Spinhoven, P., Schmahl, C., and Elzinga, B. M. (2012). Influence of emotional distraction on working memory performance in borderline personality disorder. Psychological Medicine, 42, 2181-2192.

[13] New, A. S., Goodman, M., Triebwasser, J., and Siever, L. J. (2008). Recent advances in the biological study of personality disorders. The Psychiatric Clinics of North America, 31(3), 441-461.

[14] Gray, J. R., Braver, T. S., and Raichle, M. E. (2002). Integration of emotion and cognition in the lateral prefrontal cortex. Proceedings of the National Academy of Sciences of the United States of America, 99, 41154120.

[15] Fossati, P. (2012). Neural correlates of emotion processing: From emotional to social brain. European Neuropsychopharmacology, 22, 487-491.

[16] Ray, R. D., and Zald, D. H. (2012). Anatomical insights into the interaction of emotion and cognition in the prefrontal cortex. Neuroscience and Biobehavioural Reviews, 36, 479-501.

[17] Villringer, A., and Dirnagl, U. (1995). Coupling of brain activity and cerebral blood flow: basis of functional neuroimaging. Cerebrovascular and Brain Metabolism Reviews, 7(3), 240-276.

[18] Cui, X., Bray, S., Bryant, D. M., Glover, G. H., and Reiss, A. L. (2011). A quantitative comparison of NIRS and fMRI across multiple cognitive tasks. Neuroimage, 54(4), 2808-2821.

[19] Doi, H., Nishitani, S., and Shinohara, K. (2013). NIRS as a tool for assaying emotional function in the prefrontal cortex. Frontiers in Human Neurosciences, 7(770), 1-6.

[20] Bendall, R. C. A., and Thompson, C. (2015). Functional near-infrared spectroscopy: An emerging neuroimaging tool successful in studying the interaction between emotion and cognition. PsyPAG Quarterly, 96, 1417.

[21] Kreplin, U., and Fairclough, S. H. (2013). Activation of the rostromedial prefrontal cortex during the experience of positive emotion in the context of esthetic experience. A fNIRS study. Frontiers in Human Neuroscience, 7(879), 1-7.

[22] Bruyneel, L., Stennbergen, H. V., Hommel, B., Band, G. P. H., and Raedt, R. D., and Koster, E. H. W. (2013). Happy but still focussed: failures to find evidence for a mood-induced widening of visual attention. Psychological Research, 77, 320-332.

[23] Glotzbach, E., Muhlberger, A., Gschwendtner, K., Fallgatter, A. J., Pauli, P., and Herrmann, M. J. (2011). Prefrontal brain activation during emotional processing: A functional near infrared spectroscopy study (fNIRS). The Open Nueorimaging Journal, 5, 33-39.

[24] Jasinska, A. J., Yasuda, M., Rhodes, R. E., Wang, C., and Polk, T. A. (2012). Task difficulty modulates the impact of emotional stimuli on neural responses in cognitive-control regions. Fronteris in Psychology, 3(345), 1-12.

[25] Rensink, R. A., O'regan, J. K., and Clark, J. J. (1997). To see or not to see: The need for attention to perceive change in scenes. Psychological Science, 8(5), 368-373.

[26] Lang, P. J., Bradley, M. M., and Cuthbert, B. N. (2008). International affective picture system (IAPS): Affective ratings of pictures and instruction manual. Technical Report A-8. University of Florida, Gainesville, FL.

[27] Watson, D., Clark, L. A., and Tellegen, A. (1988). Development and validation of brief measures of positive and negative affect. The PANAS scales. Journal of Psychology, 54(6), 1063-1070.

[28] Jasper, H. H. (1958). The ten-twenty electrode system of the International Federation. Electroencephalography and Clinical Neurophysiology, 10, 367-380.

[29] Ayaz, H., Izzetoglu, M., Shewokis, P. A., and Onaral, B. (2010). Sliding-window motion artefact rejection for functional near-infrared spectroscopy. Conference Proceedings IEEE Eng Med Biol. Soc, 65676570 . 
[30] Ayaz, H. (2010). Functional near infrared spectroscopy based brain computer interface. PhD Thesis, Drexel University, Philadelphia, PA.

[31] Sassaroli, A., and Fantini, S. (2004). Comment on the modified BeerLambert law for scattering media. Physics in Medicine and Biology, 49, N255-257.

[32] Herff, C., Heger, ., Fortmann, O., Hennrich, J., Putze, F., and Schultz, T. (2014). Mental workload during n-back task - quantified in the prefrontal cortex using fNIRS. Frontiers in Human Neuroscience, 7(935), 1-9.

[33] Fishburn, F. A., Norr, M. E., Medvedev, A. V., and Vaidya, C. J. (2014) Sensitivity of fNIRS to cognitive state and load. Frontiers in Human Neuroscience, 8(76), 1-11.

[34] Parasurman, R., and Giambra, L. (1991). Skill development in vigilance: effects of event rate and age. Psychological Aging, 6, 155-169.

[35] Cremers, H. R., Demenescu, L. R., Aleman, A., Renken, R., van Tol, M., van der Wee, N. J. A., ... Roelofs, K. (2010). Neuroticism modulates amygdala-prefrontal connectivity in response to negative emotional facial expressions. Neuroimage, 49, 963-970.

[36] Hahn, S., Buttaccio, D. R., Hahn, J., and Lee, T. (2015). Personality and attention: Levels of neuroticims and extraversion can predict attentional performance during a change detection task. Quarterly Journal of Experimental Psychology, 68, 1041-1048.

[37] Crocker, L. D., Heller, W., Speilberg, J. M., Warren, S., L., Bredemeier, K., Sutton, B. P., ... Miller, G. A. (2012). Neural mechanisms of attentional control differentiate trait and state negative affect. Frontiers in Psychology, 3(298), 1-13.

[38] Wang, L., Paul, N., Stanton, S. J., Greeson, J. M., and Smoski, M. J. (2013). Loss of sustained activity in the ventromedial prefrontal cortex in response to repeated stress in individuals with early-life emotional abuse: Implications for depression vulnerability. Frontiers in Psychology, 4(320), 1-9.

[39] Sanchez, T. A., Mocaiber, I., Erthal, F. S., Joffily, M., Volchan, E., Pereira, M. G., de Araujo, D. B., and Oliveria, L. (2015). Amygdala responses to unpleasnant pictures are influenced by task demands and positive affect trait. Frontiers in Human Neuroscience, 9(107), 1-9.

[40] Dolcos, F., Iordan, A. D., Kragel, J., Stokes, J., Campbell, R., McCarthy, G., and Cabeza, R. (2013). Neural correlates of opposing effects of emotional distraction on working memory and episodic memory: an event-related fMRI investigation. Frontiers in Psychology, 4(293), 1-16. 